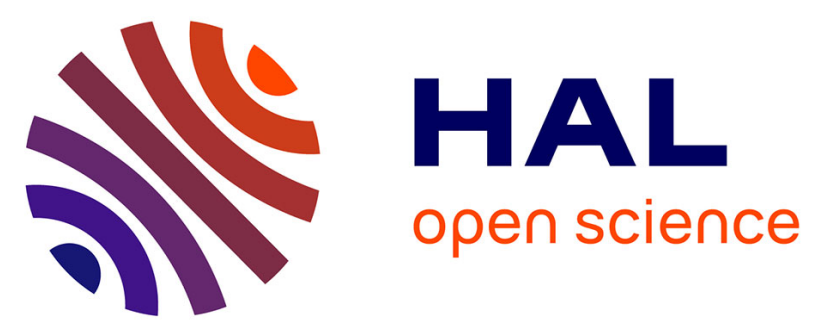

\title{
Intravenous and intratumoral injection of Pluronic P94: The effect of administration route on biodistribution and tumor retention
}

Costanza Santini, Alexandra G Arranja, Antonia G Denkova, François Schosseler, Karolina Morawska, Peter Dubruel, Eduardo Mendes, Marion de Jong, Monique R Bernsen

\section{To cite this version:}

Costanza Santini, Alexandra G Arranja, Antonia G Denkova, François Schosseler, Karolina Morawska, et al.. Intravenous and intratumoral injection of Pluronic P94: The effect of administration route on biodistribution and tumor retention. Nanomedicine: Nanotechnology, Biology and Medicine, 2017, 13 (7), pp.2179 - 2188. 10.1016/j.nano.2017.04.015 . hal-03508639

\section{HAL Id: hal-03508639 \\ https://hal.science/hal-03508639}

Submitted on 14 Jan 2022

HAL is a multi-disciplinary open access archive for the deposit and dissemination of scientific research documents, whether they are published or not. The documents may come from teaching and research institutions in France or abroad, or from public or private research centers.
L'archive ouverte pluridisciplinaire HAL, est destinée au dépôt et à la diffusion de documents scientifiques de niveau recherche, publiés ou non, émanant des établissements d'enseignement et de recherche français ou étrangers, des laboratoires publics ou privés. 


\title{
Injection route determines biodistribution and tumor accumulation of Pluronic copolymers
}

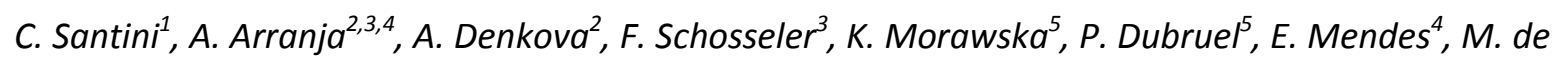
Jong $^{1}$, M. Bernsen ${ }^{1}$

* Correspondence: c.santini@erasmusmc.nl

${ }^{1}$ Department of Radiology and Nuclear Medicine, Erasmus MC, 's-Gravendijkwal 230, 3015 CE Rotterdam

${ }^{2}$ Department of Radiation Science and Technology, Delft University of Technology, Mekelweg 15, 2629 JB Delft, the Netherlands

${ }^{3}$ Institute Charles Sadron (CNRS), Strasbourg, France

${ }^{4}$ Department of Chemical Engineering, Delft University of Technology, van der Maasweg 9, $2629 \mathrm{HZ}$, 2628 BL Delft, The Netherlands

${ }^{5}$ Department of Organic and Macromolecular Chemistry, Ghent University, B-9000 Ghent, Belgium

\begin{abstract}
Introduction: Recent studies have shown that Pluronic P94 triblock copolymers display prolonged blood circulation and high tumor cell internalization, suggesting a great potential for tumor drug delivery applications. The most appropriate administration route should be determined in order to reduce undesired off-target accumulation and to reach the highest tumor uptake. In this study, the biodistribution and tumor accumulation of radiolabeled Pluronic P94 triblock copolymers following intravenous (IV) or intratumoral (IT) injections have been compared.
\end{abstract}


Methods and Materials: The P94 copolymer was modified with a radiochelator (DTPA) and radiolabeled with the gamma-emitting radionuclide ${ }^{111} \mathrm{In}$. P94- ${ }^{111} \mathrm{In}$-DPTA was administered in tumorbearing mice IV or IT, subsequently the animals underwent SPECT/CT imaging at different time points post injection (pi). The retention of gamma radiation in selected organs of interest was determined at 48,72 or $120 \mathrm{~h} \mathrm{pi}$.

Results: IV administration of P94- ${ }^{111}$ In-DPTA resulted in low tumor accumulation of radioactivity and relatively high accumulation in off-target organs such as the liver. In contrast, after IT administration, SPECT/CT scans revealed that most of the P94- ${ }^{111}$ In-DPTA copolymers remained concentrated in the tumor region with limited off-target tissue accumulation. Independent from the route of administration, the retention time of tumor radioactivity was prolonged, whereas radioactivity in other tissue was rapidly.

Conclusion: IV injection of P94- ${ }^{111}$ In-DPTA resulted in limited tumor uptake, whereas IT administration substantially improved tumor radioactivity and visualization during imaging. The minimal off-target tissue involvement, and the tracer retention over time (especially after IT administration), confirmed the potential of Pluronic P94 copolymer to be used as a carrier for intratumoral radionuclide therapy. 


\section{Introduction}

The need for specific and more efficient cancer treatments is well recognized and many efforts are being made towards the development of safe and more effective therapeutic approaches. Specially, the field of nanomedicine has grown considerably in the last decades for this purpose. Nanomedicines, represented by compounds generally ranging from 1 to $100 \mathrm{~nm}$, are commonly administered through intravenous injection (IV) in order to ensure a complete distribution over the whole body and to reach the primary tumor and metastases. After entry into the circulation, nanoparticles can then accumulate in the tumor tissue profiting from a passive phenomenon known as the Enhanced Permeability and Retention (EPR) effect (1): The leaky tumor vasculature together with reduced lymphatic drainage allow for passive extravasation and specific tumor accumulation of nano-sized medicines. Because of the passive nature of the EPR effect, nanomedicines do not require specific targeting agents and can accumulate in permeable tumors, such as sarcomas (2), head and neck tumors (3), and glioblastomas (4)

The extent of accumulation and retention of these nano compounds in the tumors has shown to vary extensively, as it depends on many parameters such as particle's properties (e.g. size, shape and charge) (5) and tumor microenvironment (e.g. tumor internal pressure, blood flow) $(6,7)$. When administered IV, high tumor uptake is generally obtained for those particles that can bypass the organism's clearance mechanisms (mainly represented by the kidneys, liver and spleen) (8) presenting long circulation times.

In order to improve tumor uptake and to reduce possible side effects, nanomedicines can be applied intratumorally (IT) by injecting the compounds directly at the tumor site. This method can be applied for those solid tumors that are not suitable for surgery, such as brain, head and neck tumors, or inoperable solid tumors (10-12). The injection in loco naturally improves the amount of tracer in the tumor. Moreover, if the compounds together with the radionuclide are retained in the tumor, the IT 
route avoids the involvement of the systemic circulation evading the main clearance mechanisms and reducing accumulation in off-target tissues. Therefore, using the IT route for the delivery of nanotherapeutics is beneficial for the safety and efficacy of the treatment of localized solid tumors $(13,14)$.

Among nanoparticles, polymers and Pluronic copolymers in particular have raised considerable interest due to their versatility and valuable properties. These polymer chains can be easily modified with different agents for imaging applications, but they can also be combined with drugs becoming especially suitable for theranostic purposes. Moreover, certain compositions of Pluronic copolymers have shown a typical biological activity such as the capacity to sensitize multi-drug resistant cancer cells increasing therefore the cytotoxic potential of chemotherapeutic drugs $(15,16)$.

Pluronic P94 is an amphiphilic linear triblock copolymer of poly(ethylene oxide)-poly(propylene oxide)-poly(ethylene oxide) (PEO-PPO-PEO) and has shown interesting properties in vitro $(17,18)$ as well as in vivo (19) for imaging and therapeutic applications. In preclinical settings, Pluronic P94 has shown to be internalized efficiently by tumor cells, with reduced exocytosis when compared with other formulations, such as Pluronic F127. It has also demonstrated to be able to disaggregate tumor spheroids in vitro (18), suggesting potentially relevant therapeutic effects. In vivo studies performed with ${ }^{111}$ In radiolabeled P94 copolymers in healthy mice have shown high in vivo stability and relatively long circulation time, while acute whole body toxicity was not reported (19). These results suggest that Pluronic P94 copolymer might be suitable for imaging and/or therapeutic applications. In this study, we applied ${ }^{111}$ In-radiolabeled P94 copolymer in tumor-bearing mice via IV and IT injection routes to evaluate for the first time the in vivo potential of Pluronic P94 triblock copolymer regarding tumor accumulation and retention. 


\section{Materials and Methods}

\subsection{Functionalization of Pluronic P94 copolymer with DTPA chelator (P94-DTPA)}

Pluronic P94 (PEO ${ }_{26} \mathrm{PPO}_{48} \mathrm{PEO}_{26} ; \mathrm{M}_{n}$ PEO blocks: $2200 \mathrm{Da} ; \mathrm{M}_{\mathrm{n}}$ PPO block: $\left.2800 \mathrm{Da}\right)$ were supplied by BASF and end-functionalized with the chelator DTPA following procedures described previously (19-21).

Briefly, P94 unimers were purified from low molecular weight contaminants (22) and functionalized with 1,1-carbodiimidazole (CDI-Pluronic) and primary amines (Pluronic- $\mathrm{NH}_{2}$ ) (19). The modified chelator p-SCN-Bn-DTPA was then conjugated to the amine-terminated copolymers. The yield of the different chemical intermediates was determined by ${ }^{1} \mathrm{H}-\mathrm{NMR}$ (Bruker WH $300 \mathrm{MHz}$ instrument) and is reported elsewhere (19).

\subsection{Radiolabeling of P94-DTPA with ${ }^{111}$ In (P94-DTPA $-{ }^{111}$ In) and radiolabeling efficiency} The radiolabeling of P94-DTPA with ${ }^{111}$ In was performed at neutral $\mathrm{pH}$ and at room temperature for 30 minutes. A stock solution of P94-DTPA $(8 \mu \mathrm{L})$ was added to a solution containing $1800 \mu \mathrm{L}$ of $1 \mathrm{X}$ PBS and $200 \mu \mathrm{L}$ of $\left.{ }^{111} \operatorname{InCl}\right|_{3}(\sim 150 \mathrm{MBq}) \cdot{ }^{111} \mathrm{InCl}_{3}$ was obtained from Mallinckrodt Pharmaceuticals (Petten, The Netherlands). The final concentration of P94-DTPA was $1 \mathrm{mg} / \mathrm{mL}$, with a specific activity of $75 \mathrm{MBq} / \mathrm{mL}$. The efficiency of the labeling reaction was analyzed by instant thin layer chromatography (iTLC) in aluminum sheets of Silica gel 60 (Merck Millipore) and EDTA/NH ${ }_{4} A c(1: 1)$ buffer $\left(R_{f}\right.$ P94-DTPA- ${ }^{111} \ln =0 ; R_{f}$ free ${ }^{111} \ln =1 ; R_{f} p$ SCN-Bn-DTPA- ${ }^{111}$ In $=0.5-1$ ) as described previously (19). For negative control studies, free DTPA was labeled with ${ }^{111}$ In $(200 \mathrm{pmol}, 3 \mathrm{MBq})\left({ }^{111} \mathrm{In}\right.$-DTPA) following the same procedure already used for labeling the DTPA-P94 polymer. Labeling efficiency was assessed with iTLC chromatography (23) on aluminum paper (TLC Aluminum Sheets, EMD/Merck KGaA).

\subsection{Tumor and animal model}


Human small cell lung cancer, H69 (ECACC, Salisbury, UK), were cultured under standard conditions in Gibco's RPMI medium (Invitrogen Corp., Breda, The Netherlands) supplemented with $10 \% \mathrm{v} / \mathrm{v}$ fetal bovine serum and $1 \% \mathrm{v} / \mathrm{v}$ penicillin/streptomycin. Cells were suspended in a 1:2 v/v Seligmann's buffered salt solution (SBSS) medium and Matrigel (BD Biosciences, Mississauga, Canada). The suspension was diluted at a dose of $100 \mu \mathrm{L} /$ mouse $\left(\sim 3 \times 10^{6}\right.$ cell/mouse) and inoculated subcutaneously in 20 young adult male Balb/c-nu mice (Janvier, France). Tumor growth was monitored and measured with a caliper ruler three times per week. All animal experiments were performed in accordance with Dutch animal welfare regulations and approved by the local ethics committee

\subsection{In vivo studies}

When the average tumor volume reached $300 \mathrm{~mm}^{3}$, the mice were randomly subdivided in three groups: one for intravenous injection (IV) ( $N=8)$ studies, one for intratumoral injection (IT) $(N=8)$ studies and third one for IT negative studies $(N=4)$. Mice from the IV group $(N=8)$ received an injection of $\sim 200 \mu \mathrm{L}(\sim 0.2 \mathrm{mg}$ and $\sim 15 \mathrm{MBq} /$ animal) in the tail vein.Mice from the IT group ( $\mathrm{N}=8)$ received $\sim 100 \mu \mathrm{L}(\sim 0.1 \mathrm{mg}$ and $\sim 7.5 \mathrm{MBq} /$ animal) in one single injection in the center of the tumor mass. The 4 mice of the IT negative control group were injected IT with $100 \mu \mathrm{L}$ of ${ }^{111} \mathrm{In}$-DTPA (200 pmol and $\sim 3 \mathrm{MBq}$ ).

\subsection{In vivo imaging SPECT/CT and biodistribution}

After the injection, mice were anesthetized with a gas mixture of isoflurane (Pharmachemie, Haarlem, The Netherlands) (4\% induction, $2 \%$ maintenance) and oxygen and subjected to whole body and focus SPECT/CT static scan at different time points. Six mice from the IV group and six mice from the IT group were randomly chosen and further subjected to SPECT/CT scan. Nuclear images were acquired using the U-SPECT-II system (MiLabs B.V., Utrecht, The Netherlands) equipped with the new ultra-high-sensitivity collimator Mouse 2.0, having 54 
conical pinholes of $2.0 \mathrm{~mm}$ diameter (24). The U-SPECT-II system has a heating system to prevent the hypothermia of the animals during the scan. The acquisition time of each scan was approximately 36 minutes for the whole body (40-42 positions, $52 \mathrm{sec} /$ position) and $15 \mathrm{~min}$ (14 positions, $66 \mathrm{sec} /$ position) for the focus scan. A CT scan (240 projections, $500 \mathrm{~ms}$ exposure time $55 \mathrm{kVp}$ tube voltage) was also performed for anatomical reference. The focus SPECT/CT scan was performed at $0.5 \mathrm{~h}$ post injection (pi) in IT injected mice. Whole body SPECT/CT images were acquire at early time points ( $4 \mathrm{~h} \mathrm{pi})$, early-intermediate ( $24 \mathrm{~h} \mathrm{pi)}$ and intermediate (48 $\mathrm{h} \mathrm{pi).} \mathrm{Three} \mathrm{mice} \mathrm{from} \mathrm{the} \mathrm{IT} \mathrm{injection} \mathrm{and} \mathrm{three} \mathrm{mice} \mathrm{from} \mathrm{IV} \mathrm{injection} \mathrm{group} \mathrm{were}$ randomly chosen and further subjected to later time points of $72 \mathrm{~h}$ and $120 \mathrm{~h}$ pi respectively. Biodistribution studies were performed at intermediate ( $48 \mathrm{~h}$ pi) and at late time points (72 or $120 \mathrm{~h}$ pi) (Fig 2). After the corresponding SPECT/CT scans, mice were sacrificed by cervical dislocation, and blood along with a selection of organs were collected. The radiation arising from the different organs was measured with the aid of a gamma counter (Wallac, 1480 Wizard 3", PerkinElmer, Groningen, The Netherlands). For each tissue, the polymer uptake was calculated as a percentage of the injected dose per gram of tissue (\% ID/g).

\subsection{Images processing and quantification}

SPECT/CT images were reconstructed using dedicated software Posem [1] algorithm (25). Based on the acquired images, the uptake and accumulation of P94 radiolabeled copolymers after IV and IT injection was monitored in selected organs (heart, bladder, tumor, liver, lungs, liver) by drawing a region of interest around the specific organ based on CT images. The quantification was performed at 4, 24, 48 and $120 \mathrm{~h}$ after IV injection and at 24, 48, and $72 \mathrm{~h}$ after IT injection. The intensity of signal per unit of tissue was measured and the uptake calculated as a percentage of the injected activity $\% \mathrm{ID} / \mathrm{mm}^{3}$. The quantification of images was performed using VivoQuant iPACS Software Version 1.23 (INVICRO, Boston, MA, USA). 


\subsection{Software and statistical analyses}

All the data collected were processed with Microsoft Excel 2010 and Prism version 5 (GraphPad Software, La Jolla, California, USA). Data from the in vivo evaluation were analyzed using either exponential fitting or linear regression. Ex vivo based biodistribution results were analyzed with Mann-Whitney $U$ test, setting $p<0.05$ as significantly different. Outliers were determined using the modified Thompson Tau Technique.

\section{Results}

3.1. Functionalization of P94 triblock copolymers with DTPA chelator and radiolabeling (P94- ${ }^{111}$ InDTPA)

Pluronic P94 triblock copolymers were purified from low molecular weight contaminants resulting in compounds with high purity (98\%) (22). These were functionalized with the chelator DTPA and successfully radiolabeled with the radionuclide ${ }^{111}$ In using standard conditions, reaching a labeling efficiency of $>91 \%$. Because of the good yield, no further purification of the compound was needed (Fig 1). 
A)

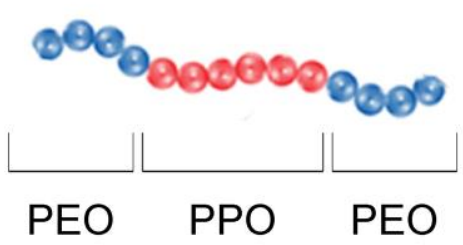

P94

B)

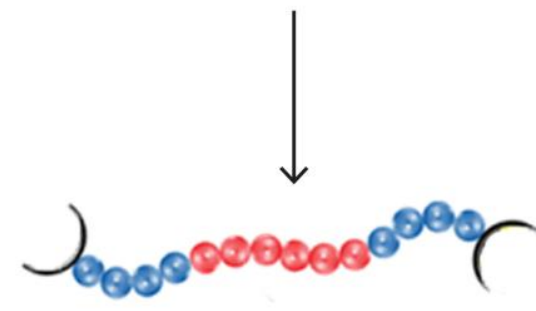

P94-DTPA

C)

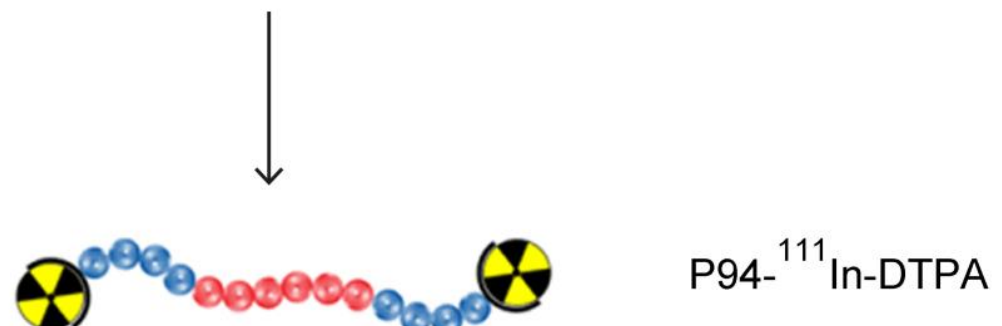

Fig 1. Schematic representation of the successive modifications of Pluronic P94 triblock copolymer $\left.\left(\mathrm{PEO}_{26} \mathrm{PPO}_{48} \mathrm{PEO}_{26}\right)\right)$. A) Plain polymer (P94); B) Attachment of DTPA chelator (P94-DTPA); C) Radiolabeling with ${ }^{111}$ In (P94- ${ }^{111}$ In-DTPA). PEO: poly(ethyleneoxyde); PPO: poly(propylene oxide); DTPA: diethylenetriaminepentaacetic acid

\subsection{SPECT/CT imaging and in vivo quantification}

After tumor inoculation and IV or IT injections of radiolabeled Pluronic P94 copolymers (Fig. 2), SPECT/CT scans of the total body distribution of radioactivity were obtained. in parallel, a negative control group injected intratumorally with ${ }^{111}$ In-DTPA revealed a very fast clearance of the compound from the tumor tissue at the first time point $(0.5 \mathrm{~h})$ (Supplementary information). 


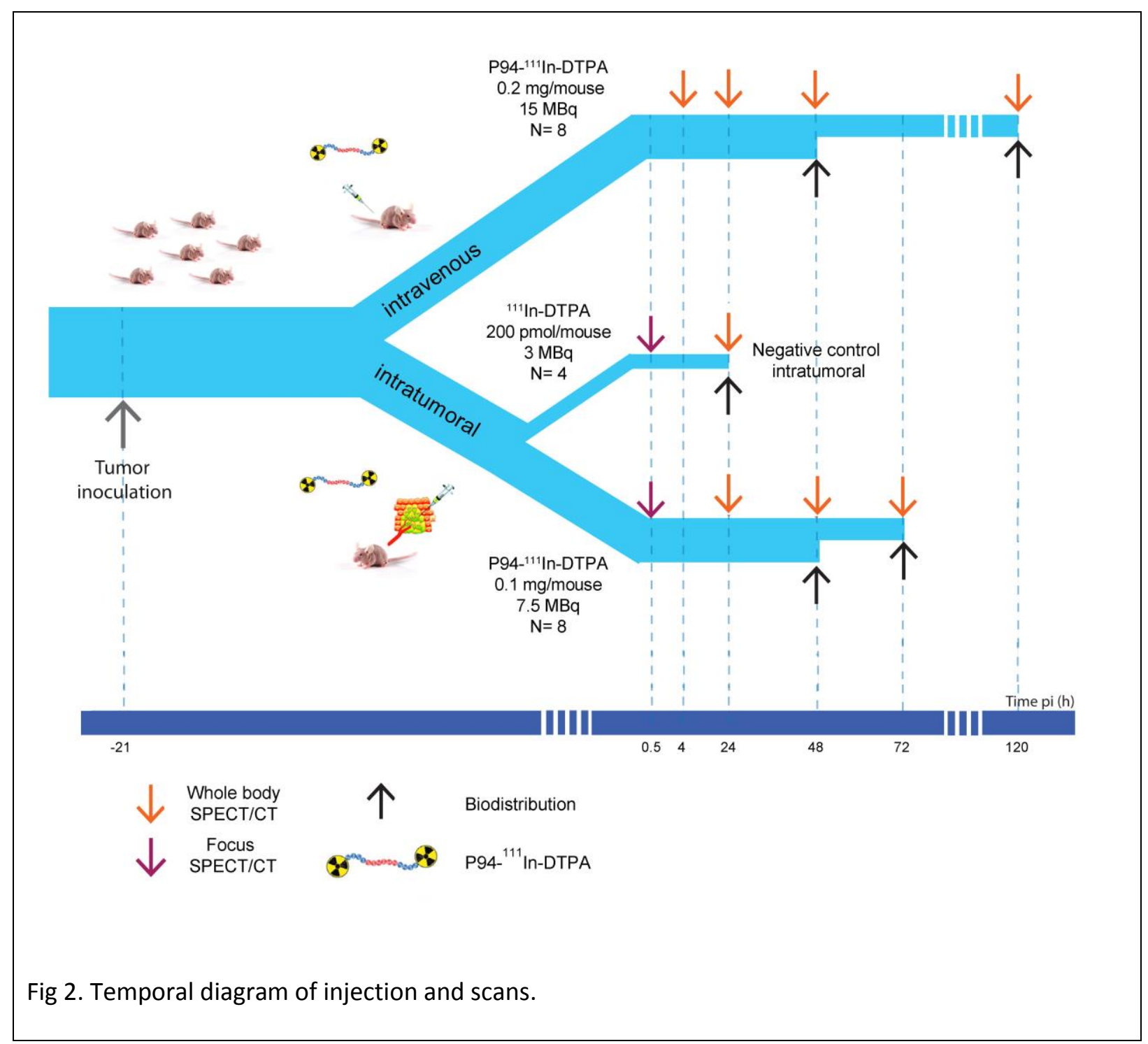

After IV injection of the radiolabeled copolymers, we observed at the first time point ( $4 \mathrm{~h} \mathrm{pi)}$ a substantial accumulation of the copolymers in the bladder, which is associated to the fact that these nano-sized compounds $(\sim 4 \mathrm{~nm})$ are below the renal clearance cut-off as observed previously (19). At the same time point, we also observed a sustained accumulation in the liver with minor involvement of the tumor (Fig 3; Table 1). The polymer uptake in the offtarget tissues was relatively high $\left(>0.1 \mathrm{kBq} / \mathrm{mm}^{3}\right)$ as observed by the elevated accumulation in the heart, lungs and kidney (Table 1). However, we observed an overall decrease of activity in all organs indicating that these compounds are not retained over time and follow a negative exponential trend, including the tumor (Fig 3B). 
Table 1. Accumulation of P94- ${ }^{111}$ In-DTPA in selected organs at different time points, after intravenous injection (IV). sd: standard deviation.

\begin{tabular}{|c|cc|cc|cc|cc||}
\hline \multirow{2}{*}{} & \multicolumn{2}{|c|}{$4 \mathrm{~h}$} & \multicolumn{2}{c|}{$24 \mathrm{~h}$} & \multicolumn{2}{c|}{$48 \mathrm{~h}$} & \multicolumn{2}{c|}{$120 \mathrm{~h}$} \\
\hline KBq/mm & mean & sd & mean & sd & mean & sd & mean & sd \\
\hline Bladder & 0,618 & 0,102 & 0,060 & 0,014 & 0,032 & 0,003 & 0,041 & 0,025 \\
Heart & 0,183 & 0,015 & 0,080 & 0,023 & 0,035 & 0,004 & 0,02 & 0,005 \\
Liver & 0,360 & 0,011 & 0,308 & 0,031 & 0,270 & 0,044 & 0,190 & 0,023 \\
Lungs & 0,141 & 0,008 & 0,062 & 0,012 & 0,041 & 0,015 & 0,022 & 0,004 \\
Kideny & 0,122 & 0,022 & 0,069 & 0,015 & 0,050 & 0,012 & 0,033 & 0,006 \\
Tumor & 0,053 & 0,003 & 0,039 & 0,010 & 0,032 & 0,008 & 0,018 & 0,001 \\
\hline
\end{tabular}




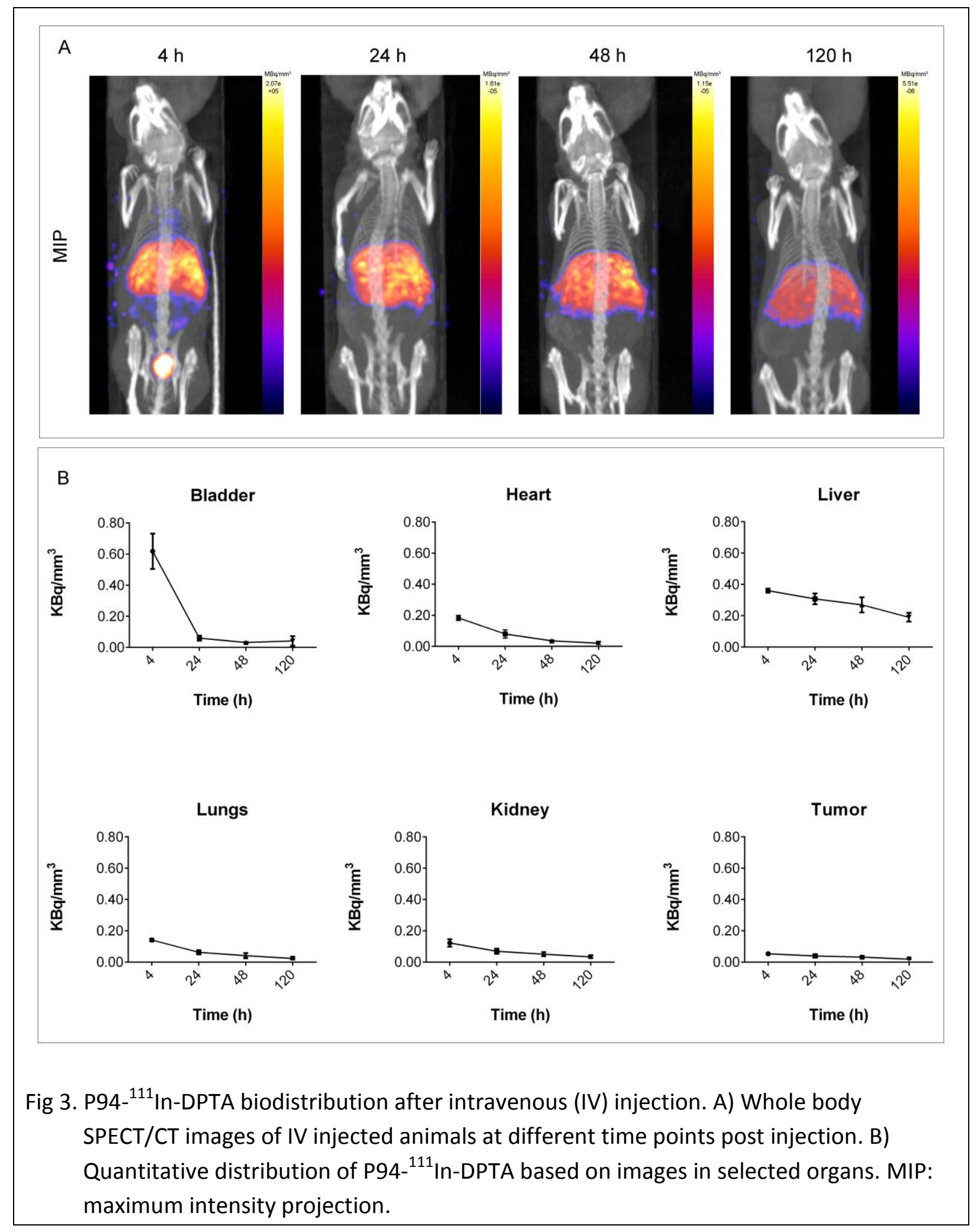

After IT injection, radioactivity could be measured only in the tumor and, at later time points, in the liver (Fig 4; Table 2). In the tumor, SPECT/CT scans showed that the localization of the P94 copolymers changed over time (Fig 4B-C). Immediately following injection, the 
distribution in the tumor was homogeneous whereas, at later time points ( $\sim 24 \mathrm{~h}$ ), the results indicated that the radioactivity migrated to the tumor margins, where it remained at least up to $72 \mathrm{~h}$ pi (Fig 4B). The amount of radioactivity detected in the tumor after IT injection was markedly constant over time (Table 2). Also, for the other selected organs, the amount of radioactivity measured between 24 and $72 \mathrm{~h}$ was low and fairly constant (Fig $4 \mathrm{C}$ ).

Table 2. Accumulation of P94- ${ }^{111}$ In-DTPA in selected organs at different time points after intratumoral (IT) injection. sd: standard deviation; *: not available.

\begin{tabular}{|c|cc|cc|cc|cc|}
\hline \multicolumn{1}{c|}{} & \multicolumn{2}{|c}{$0,5 \mathrm{~h}$} & \multicolumn{2}{c|}{$24 \mathrm{~h}$} & \multicolumn{2}{c|}{$48 \mathrm{~h}$} & \multicolumn{2}{c|}{$72 \mathrm{~h}$} \\
\hline $\mathrm{KBq} / \mathrm{mm}^{3}$ & mean & sd & mean & sd & mean & sd & mean & sd \\
\hline Bladder & $*$ & $*$ & 0,035 & 0,021 & 0,019 & 0,007 & 0,019 & 0,004 \\
Heart & $*$ & $*$ & 0,045 & 0,011 & 0,041 & 0,017 & 0,023 & 0,005 \\
Liver & $*$ & $*$ & 0,126 & 0,021 & 0,126 & 0,027 & 0,120 & 0,010 \\
Lungs & $*$ & $*$ & 0,046 & 0,015 & 0,049 & 0,015 & 0,028 & 0,004 \\
Kideny & $*$ & $*$ & 0,050 & 0,016 & 0,043 & 0,015 & 0,049 & 0,004 \\
Tumor & $*$ & $*$ & 0,662 & 0,105 & 0,541 & 0,049 & 0,493 & 0,059 \\
\hline
\end{tabular}




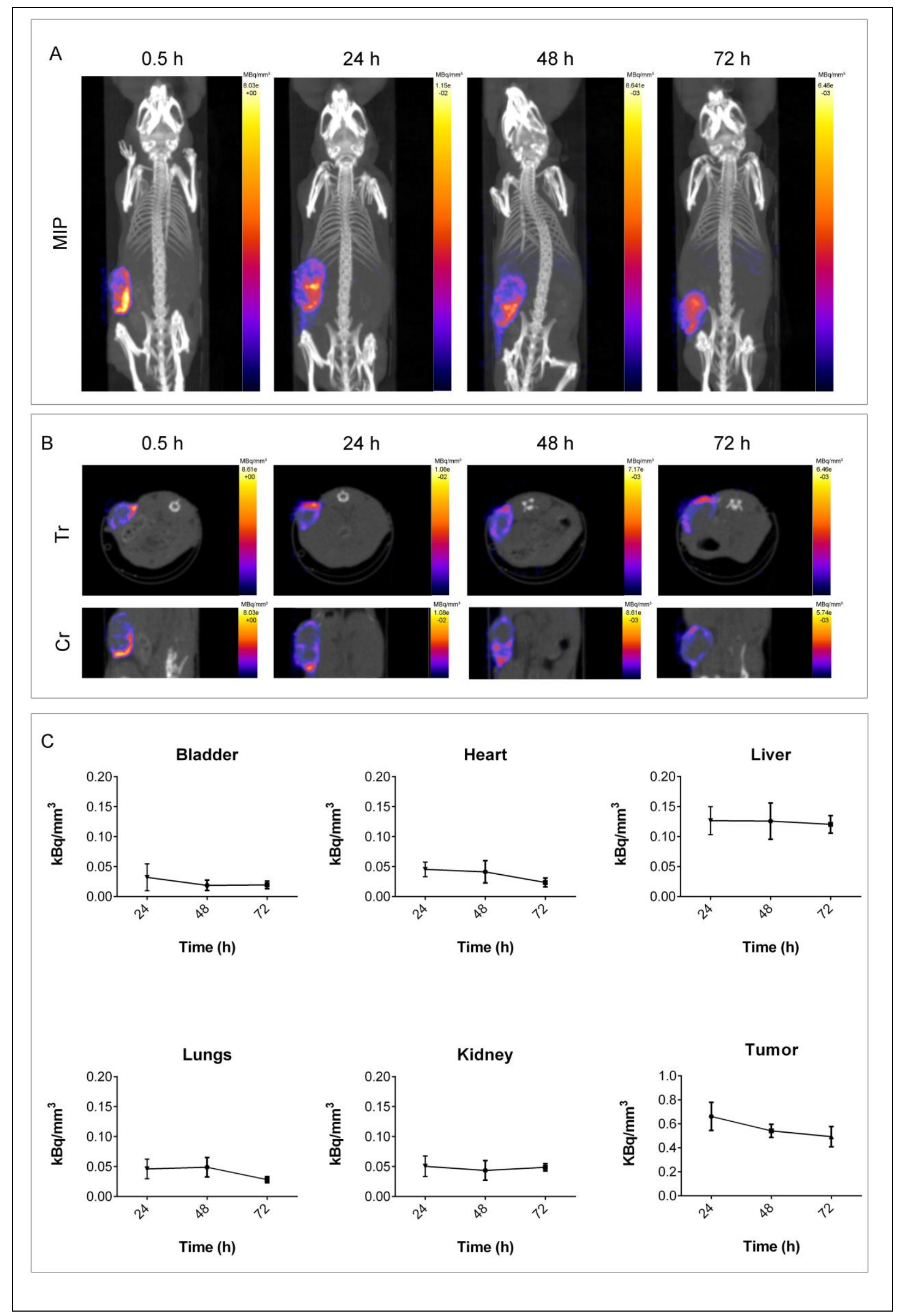


Fig 4. P94- ${ }^{111}$ In-DPTA biodistribution after intratumoral (IT) injection. A) Whole body SPECT/CT images of IT injected animals at different time points post injection. B) SPECT/CT images of tumor region, at different time points post injection. C) Quantitative distribution of P94- ${ }^{111}$ In-DPTA based on images in selected organs. MIP: maximum intensity projection. Cr: coronal view; Tr: transversal view.

\subsection{Biodistribution results}

The uptake in the different organs following IV injection at 48 and $120 \mathrm{~h}$ pi was confirmed by performing ex vivo quantification. As expected for this type of compounds, we observed elevated uptake in the liver, spleen and kidney as these organs are involved in the clearance of polymers. The uptake in off-target tissues was relatively high being in general above $1 \% \mathrm{ID} / \mathrm{g}$, with only the muscle as an exception $(0.05 \% \mathrm{ID} / \mathrm{g})$. Especially, the liver and spleen presented the highest uptake (Table 3). As observed in the in vivo studies, quantification based on ex vivo data confirmed that Pluronic P94 copolymers uptake in the tumor after IV injection at $48 \mathrm{~h}$ and $120 \mathrm{~h}$ pi was not higher than that in any other off-target tissue (Table 3; Fig 5A).

In contrast, the biodistribution after IT injection showed a different pattern. The majority of the off-target organs presented low uptake, in general below $0.5 \% \mathrm{ID} / \mathrm{g}$ (except for skin and muscle), and a substantial tumor retention. Among the clearance organs, after IT injection only the liver presented considerable uptake (Table 3; Fig 5B), while the uptake in the spleen and the kidney was low $(<1 \% \mathrm{ID} / \mathrm{g})$ and in line with that of the other off-target tissues. As already indicated by the in vivo studies, we could confirm the elevated retention of Pluronic P94 in the tumor (Fig 5B).

The retention of radioactivity, which is directly related to the retention of the P94 copolymers, was compared between the two latest time points in all the investigated tissues. The results indicated that, independently from the injection route, in the majority of the off target tissue we observe a significant reduction $(p<0.05)$ of measured radioactivity. That behavior suggests a physiological clearance effect in the different tissues. The only exceptions to that are 
represented by the clearance organs (liver, spleen and kidney) and the skin that shows no statistical difference $(p>0.05)$ between 48 and $72 / 120 \mathrm{~h}$ pi.

The retention of radioactivity in the tumor was different from that observed in the other off target tissues. In fact, independently from the injected routes, we observed no changes in the retention in the tumor mass between the last two pi time points.

In parallel, the ex vivo evaluation of the control group confirmed the fast clearance of the ${ }^{111}$ InDTPA, with no tumor retention or accumulation in any of the investigated organs (Supplementary Information). 
A

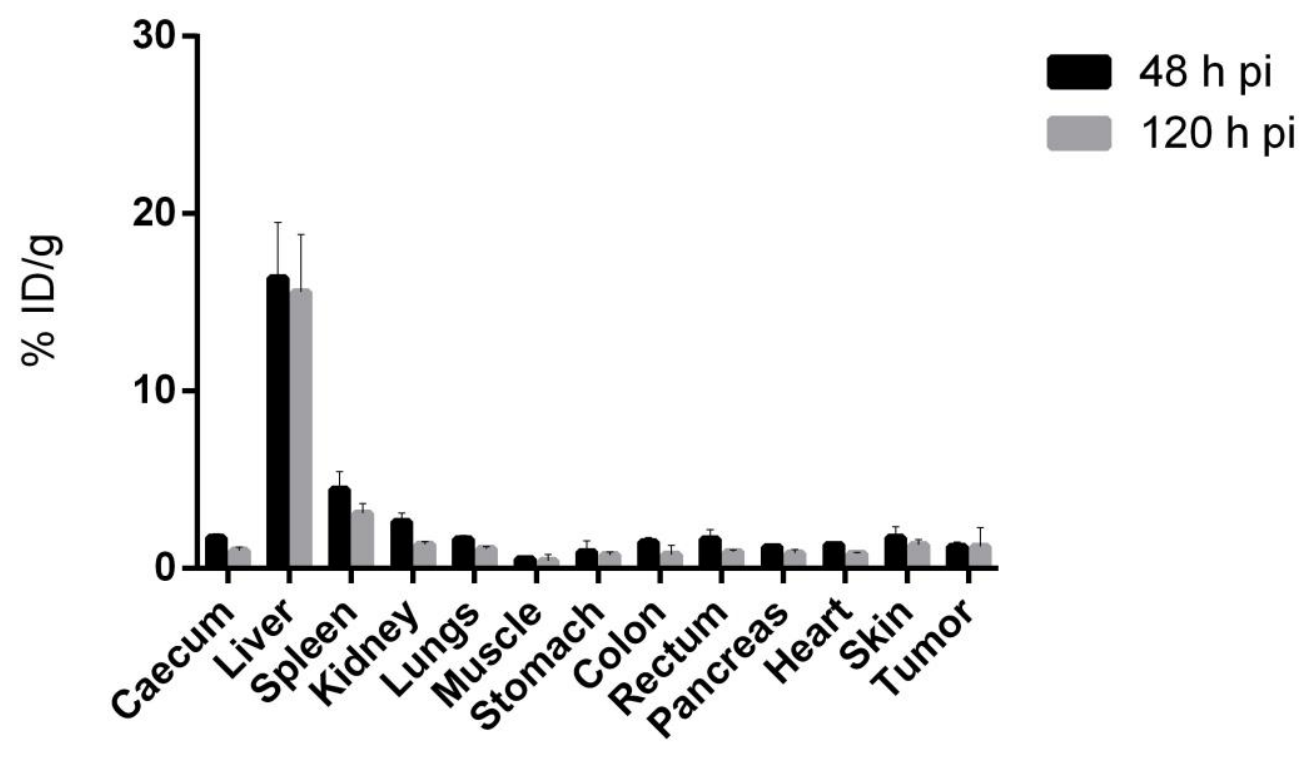

B

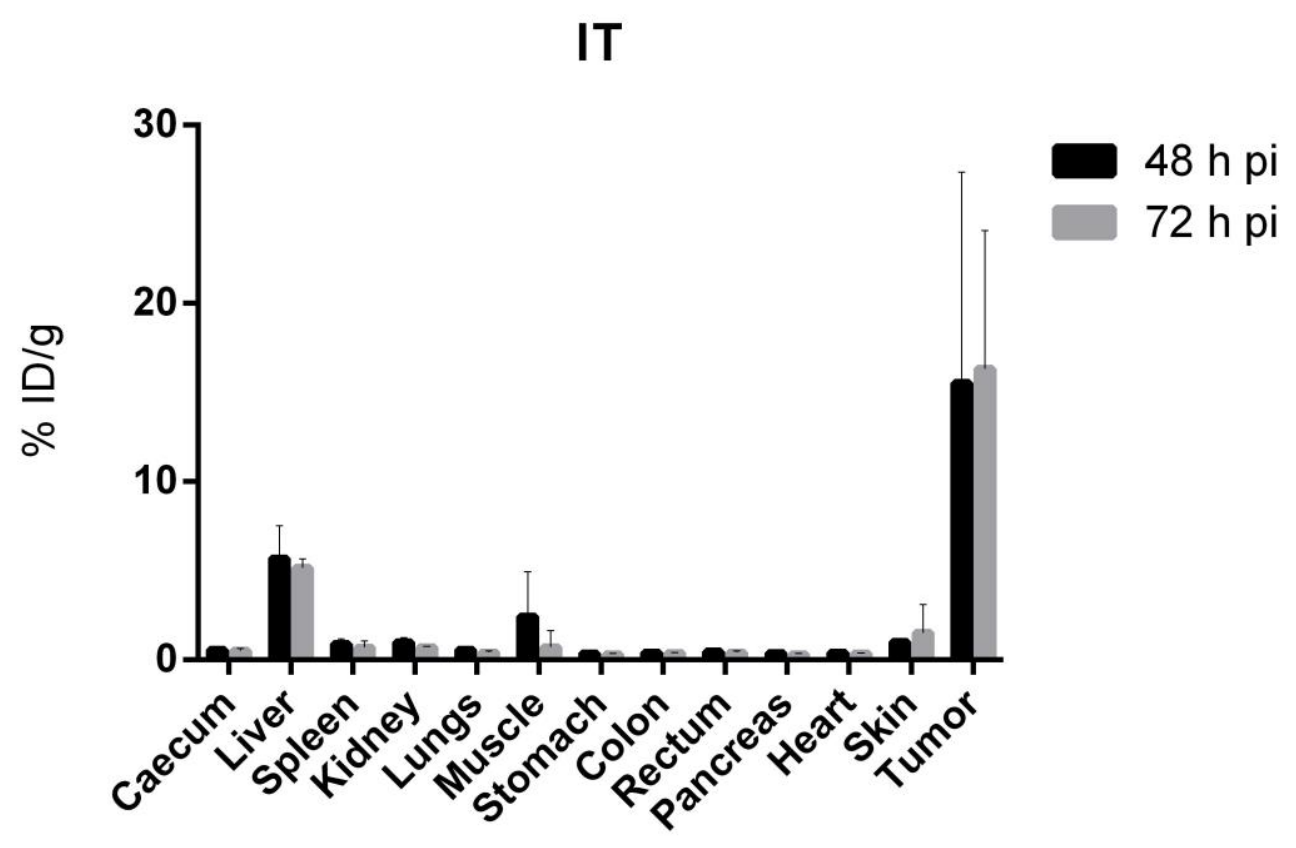

Fig 5. Biodistribution results in selected organs at 48 and 72/120 h after (A) intravenous (IV) or (B) intratumoral (IT) injection of P94- ${ }^{111}$ In-DPTA.

\section{Discussion}


Below a certain critical temperature (CMT) and concentration (CMC), Pluronic copolymers consist of free polymer chains in solution that are highly compact with the terminal PEO blocks surrounding the non-hydrated PPO block (26). In the case of the P94 triblock copolymers, in aqueous solution at $15^{\circ} \mathrm{C}$, these aggregated polymer chains present an average diameter of 4 nm (19) and a neutral zeta potential. In this work, we evaluated the potential of these nanosized polymer chains to be used for imaging and therapeutic application. The radiolabeled P94${ }^{111}$ In-DPTA triblock copolymers were administered to tumor bearing animals using two different injection routes, IV and IT, followed by SPECT/CT imaging and ex vivo quantification of the total body biodistribution over time. In clinical practice, both systemic and local injections are being used for tumor treatment $(27,28)$. The choice between the two injection routes is mainly determined by specific needs. Systemic application, such as IV injection, is the route of choice for diagnosis and also for treatment of (potentially) metastasized lesions $(27,28)$. Local (intratumor) injections on the other hand have shown beneficial outcomes for the treatment of tumors as well as they may result in higher concentrations of drugs in the tumor with limited the effects in off-target organs (28). The biodistribution results obtained after IV versus IT in vivo administration of radiolabeled Pluronic P94 copolymers confirmed a profound difference in distribution profiles and in the potential therapeutic application effect.

After IV injection, radiolabeled P94 copolymers presented limited uptake in the tumor ( $1 \%$ ID/g), which is probably resulting from the poor EPR-mediated extravasation of these nanosized triblock copolymers ( $\sim \mathrm{nm}$ ) and/or low EPR of the type of tumor used in this study. As expected, uptake in the liver, spleen and kidneys was considerable as they represent the main clearance organs for these nano compounds $(19,29-31)$. The relatively high radioactivity measured in the stomach ( $1 \% \mathrm{ID} / \mathrm{g}$ ) may result from unspecific uptake, although it can also be partially due to the phenomenon of self- and social grooming in mice (32). The uptake in the digestive tract, particularly in caecum, colon and rectum, might be due to liver and bile 
clearance $(8,33)$ as also observed previously in healthy mice after IV injection of Pluronic P94 and F127 (19), and other Pluronic copolymers $(29,30)$.

After IT injection, the P94 copolymers remained mostly confined to the tumor and reach the liver only at late time points, while the uptake in the other organs remained low. The radiolabeled P94 copolymers for this approach were administered in one single injection in the center of the tumor mass. At later time points, the re-distribution of the radioactivity to the tumoral rim indicated that there is probably a significant effect of the pressure driving fluids to the periphery of the tumor mass, thus pushing the copolymers to the tumor margins (34). This mechanism implies movement of liquid within the tumor (35), from the inside to the outside, as a consequence of the differences in hydrostatic pressure. The same mechanism might also be responsible for release of the copolymers in the systemic circulation, and ultimately for the whole body distribution as observed. However, the relatively high and long retention of the radioactivity in the tumor mass suggests that this compound is internalized by the tumor cells, as it has been observed previously in vitro as well (18).

The total body retention of P94 copolymers over time after IV injection declined gradually, indicating constant clearance of the compound. This effect was less pronounced after IT injection. Interestingly, independently from the injection route, the tumor retention between $48 \mathrm{~h}$ and the latest time point (being 120 and $72 \mathrm{~h}$ for IV and IT injection, respectively) was not statistically different meaning that the compounds were not cleared from the tumor tissue. This finding might have an important implication in view of tumor treatment potentially leading to higher therapeutic efficacy.

Regarding potential toxicity in the off-target organs, our results indicate that using either IV or IT injections, healthy tissues are inevitably involved in the uptake of P94 copolymers. Resulting 
potential off-target toxicity might be more relevant in those organs and tissues that have shown reduced clearance, such as the liver, spleen and skin. In the case of the liver, it is known that this organ has a wide-ranging resistance to drugs, as observed with other nanocarriers (36), and thus low liver toxicity is to be expected. In the case of skin and spleen, however, offtarget toxicity might be a dose-limiting factor for long term therapy as observed or suggested in previous investigation with other nanoparticles $(8,37)$.

The copolymer P94, in contrast with many other carries, can be used as a therapeutic compound or an adjuvant in chemotherapeutic treatments due to its ability to be internalized by the cells, to reach the cytoplasm and nucleus (18), and to possess cytotoxic effects at high concentrations (17). In this study, we have radiolabeled the P94 copolymers with ${ }^{111}$ In and showed its potential for imaging purposes, such as follow-up imaging of treatment progression. Because of the specific behavior of P94 copolymers once internalized by the cells (18), this carrier could also be used, for instance, to provide Auger electron therapy by selecting appropriate radionuclides (38).

We conclude that the novel Pluronic P94 nanocarrier is very promising as a drug providing toxicity to tumor cells, as an imaging agent to monitor the safety and efficacy of the therapy, and as a carrier for many different radiotherapeutics. 


\section{Conclusion}

Our results indicate radiolabeled P94 copolymers can be ligand for drugs and can be employed as carrier for drug delivery after IV and IT injection. After IV injection, the relatively long circulation time and significant uptake in healthy organs might hamper their further application and thus the right radionuclide should be carefully selected. In contrast, when administered via IT injection, P94 copolymers present extremely high and long lasting radioactivity in the tumor and minimal uptake in off-target tissues, providing excellent prospective for application as a carrier for radionuclide imaging and/or treatment.

The possibility to easily modify and radiolabel P94 with specific radionuclides suitable for imaging (i.e. ${ }^{111}$ In) and therapy suggests the potential use of radiolabeled P94 not only as (chemo)therapeutics agent itself, but also as a molecular/nuclear imaging and theranostic agent. Further studies will be focused on the therapeutic efficacy to determine the effective anticancer potency of this promising compound, and its future applicability in the clinics.

\section{Acknowledgments}

This work was supported using imaging equipment provided by the Applied Molecular Imaging Erasmus MC facility. The research leading to these results has received funding from the People Programme (Marie Curie Actions) of the European Union's Seventh Framework Programme (FP7/2007-2013) under REA grant agreement no. PITN-GA-2012-317019`TRACE ‘n TREAT. The authors would like to thank all the members of the consortium under the Marie Curie Actions, (“Trace'n'Treat") for their support, collaboration and fruitful discussions. 


\section{References}

1. Maeda H. Macromolecular therapeutics in cancer treatment: The EPR effect and beyond. Journal of Controlled Release. 2012;164:138-144.

2. Koukourakis MI, Koukouraki S, Giatromanolaki A, et al. High intratumoral accumulation of stealth liposomal doxorubicin in sarcomas--rationale for combination with radiotherapy. Acta Oncol. 2000;39:207-211.

3. Harrington KJ, Mohammadtaghi S, Uster PS, et al. Effective targeting of solid tumors in patients with locally advanced cancers by radiolabeled pegylated liposomes. Clin Cancer Res. 2001;7:243-254.

4. Koukourakis MI, Koukouraki S, Fezoulidis I, et al. High intratumoural accumulation of stealth ${ }^{\circledR}$ liposomal doxorubicin $\left(\right.$ Caelyx $\left.{ }^{\circledR}\right)$ in glioblastomas and in metastatic brain tumours. British Journal of Cancer. 2000;83:1281-1286.

5. Duan X, Li Y. Physicochemical characteristics of nanoparticles affect circulation, biodistribution, cellular internalization, and trafficking. Small. 2013;9:1521-1532.

6. Song G, Darr DB, Santos CM, et al. Effects of tumor microenvironment heterogeneity on nanoparticle disposition and efficacy in breast cancer tumor models. Clin Cancer Res. 2014;20:6083-6095.

7. Miao L, Huang L. Exploring the tumor microenvironment with nanoparticles. Cancer Treat Res. 2015;166:193-226.

8. Bertrand N, Leroux JC. The journey of a drug-carrier in the body: an anatomophysiological perspective. J Control Release. 2012;161:152-163.

9. Lammers T, Kiessling F, Hennink WE, Storm G. Drug targeting to tumors: principles, pitfalls and (pre-) clinical progress. J Control Release. 2012;161:175-187.

10. Walter KA, Tamargo RJ, Olivi A, Burger PC, Brem H. Intratumoral chemotherapy. Neurosurgery. 1995;37:1128-1145.

11. Nemunaitis J. Head and neck cancer: response to p53-based therapeutics. Head Neck. 2011;33:131-134. 
12. Celikoglu F, Celikoglu SI, York AM, Goldberg EP. Intratumoral administration of cisplatin through a bronchoscope followed by irradiation for treatment of inoperable nonsmall cell obstructive lung cancer. Lung Cancer. 2006;51:225-236.

13. Ray A, Williams MA, Meek SM, et al. A phase I study of intratumoral ipilimumab and interleukin-2 in patients with advanced melanoma. Oncotarget. 2016;6:10453.

14. Liang M, Fan $\mathrm{K}$, Zhou M, et al. H-ferritin-nanocaged doxorubicin nanoparticles specifically target and kill tumors with a single-dose injection. Proc Natl Acad Sci U S A. 2014;111:14900-14905.

15. Kabanov AV, Batrakova EV, Alakhov VY. Pluronic block copolymers as novel polymer therapeutics for drug and gene delivery. J Control Release. 2002;82:189-212.

16. Batrakova EV, Kabanov AV. Pluronic block copolymers: evolution of drug delivery concept from inert nanocarriers to biological response modifiers. J Control Release. 2008;130:98-106.

17. Arranja A, Schroder AP, Schmutz M, Waton G, Schosseler F, Mendes E. Cytotoxicity and internalization of Pluronic micelles stabilized by core cross-linking. Journal of Controlled Release. 2014;196:87-95.

18. Arranja A, Denkova AG, Morawska K, et al. Interactions of Pluronic nanocarriers with 2D and 3D cell cultures: Effects of PEO block length and aggregation state. Journal of Controlled Release. 2016;224:126-135.

19. Arranja A, Ivashchenko O, Denkova AG, et al. SPECT/CT Imaging of Pluronic Nanocarriers with Varying Poly(ethylene oxide) Block Length and Aggregation State. Mol Pharm. 2016;13:1158-1165.

20. Lu HF, Lim WS, Wang J, et al. Galactosylated PVDF membrane promotes hepatocyte attachment and functional maintenance. Biomaterials. 2003;24:4893-4903.

21. Hoang B, Lee H, Reilly RM, Allen C. Noninvasive monitoring of the fate of $111 \mathrm{In}$ labeled block copolymer micelles by high resolution and high sensitivity microSPECT/CT imaging. Mol Pharm. 2009;6:581-592.

22. Arranja A, Waton G, Schosseler F, Mendes E. Lack of a unique kinetic pathway in the growth and decay of Pluronic micelles. Soft Matter. 2016;12:769-778.

23. Chen F, Decristoforo C, Rohrbacher B, Riccabona G. A simple two-strip method to determine the radiochemical purity of technetium-99m mercaptoacetyltriglycine. European Journal of Nuclear Medicine. 1993;20:334-338. 
24. Ivashchenko O, van der Have F, Goorden MC, Ramakers RM, Beekman FJ. Ultrahigh-sensitivity submillimeter mouse SPECT. J Nucl Med. 2015;56:470-475.

25. Branderhorst W, Vastenhouw B, Beekman FJ. Pixel-based subsets for rapid multipinhole SPECT reconstruction. Phys Med Biol. 2010;55:2023-2034.

26. Alexandridis $\mathrm{P}$, Alan Hatton T. Poly(ethylene oxide) - poly(propylene oxide) $\cdot$ poly(ethylene oxide) block copolymer surfactants in aqueous solutions and at interfaces: thermodynamics, structure, dynamics, and modeling. Colloids and Surfaces A: Physicochemical and Engineering Aspects. 1995;96:1-46.

27. Kwekkeboom DJ, Krenning EP. Peptide Receptor Radionuclide Therapy in the Treatment of Neuroendocrine Tumors. Hematol Oncol Clin North Am. 2016;30:179-191.

28. Cordier D, Krolicki L, Morgenstern A, Merlo A. Targeted Radiolabeled Compounds in Glioma Therapy. Seminars in Nuclear Medicine. 2016;46:243-249.

29. Grindel JM, Jaworski T, Piraner O, Emanuele RM, Balasubramanian M. Distribution, metabolism, and excretion of a novel surface-active agent, purified poloxamer 188, in rats, dogs, and humans. J Pharm Sci. 2002;91:1936-1947.

30. Wang ZY, Stern IJ. Disposition in rats of a polyoxypropylene-polyoxyethylene copolymer used in plasma fractionation. Drug Metab Dispos. 1975;3:536-542.

31. Batrakova EV, Li S, Li Y, Alakhov VY, Elmquist WF, Kabanov AV. Distribution kinetics of a micelle-forming block copolymer Pluronic P85. J Control Release. 2004;100:389-397.

32. Blanchard RJ, McKittrick CR, Blanchard DC. Animal models of social stress: effects on behavior and brain neurochemical systems. Physiology \& Behavior. 2001;73:261-271.

33. Longmire M, Choyke PL, Kobayashi H. Clearance properties of nano-sized particles and molecules as imaging agents: considerations and caveats. Nanomedicine. 2008;3:703-717.

34. Boucher Y, Jain RK. Microvascular pressure is the principal driving force for interstitial hypertension in solid tumors: implications for vascular collapse. Cancer Res. 1992;52:5110-5114.

35. Munson JM, Shieh AC. Interstitial fluid flow in cancer: implications for disease progression and treatment. Cancer Manag Res. 2014;6:317-328.

36. Yu M, Zheng J. Clearance Pathways and Tumor Targeting of Imaging Nanoparticles. ACS Nano. 2015;9:6655-6674. 
37. Lotem M, Hubert A, Lyass O, et al. Skin toxic effects of polyethylene glycol-coated liposomal doxorubicin. Arch Dermatol. 2000;136:1475-1480.

38. Hoang B, Reilly RM, Allen C. Block Copolymer Micelles Target Auger Electron Radiotherapy to the Nucleus of HER2-Positive Breast Cancer Cells. Biomacromolecules. 2012;13:455-465. 

Supplementary

\title{
Injection route determines biodistribution and tumor accumulation of Pluronic copolymers
}

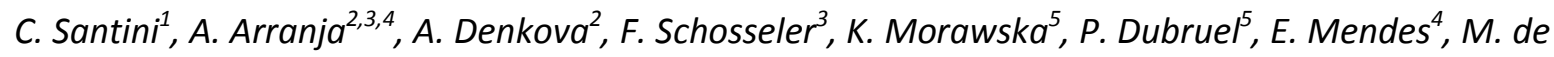 \\ Jong $^{1}$, M. Bernsen ${ }^{1}$ \\ *Correspondence: c.santini@erasmusmc.nl \\ ${ }^{1}$ Department of Radiology and Nuclear Medicine, Erasmus MC, 's-Gravendijkwal 230, 3015 CE \\ Rotterdam \\ ${ }^{2}$ Department of Radiation Science and Technology, Delft University of Technology, Mekelweg 15, \\ 2629 JB Delft, the Netherlands \\ ${ }^{3}$ Institute Charles Sadron (CNRS), Strasbourg, France \\ ${ }^{4}$ Department of Chemical Engineering, Delft University of Technology, van der Maasweg 9, $2629 \mathrm{HZ}$, \\ 2628 BL Delft,, The Netherlands \\ ${ }^{5}$ Department of Organic and Macromolecular Chemistry, Ghent University, B-9000 Ghent, Belgium
}

\section{Negative control group}

\section{Imaging and biodistribution studies}

The 4 mice chosen for negative control were injected intratumorally (IT) with ${ }^{111}$ In-DTPA subjected to SPECT/CT scan at $0.5,24$ and $48 \mathrm{~h}$ post injection and sacrificed for biodistribution purposes after the last scan.

The images acquired with a focus scan in the tumor area at $0.5 \mathrm{~h}$ pi showed a successful injection in the tumor mass, where the radioactivity is confined. At later time points, namely 24 and $48 \mathrm{~h}$, the images obtained with a whole body showed no specific retention in any tissue organs (Fig 1A-D). The biodistribution performed $48 \mathrm{~h}$ pi, confirmed the generalized limited retention of ${ }^{111}$ In-DTPA. We observe only some retention in the tumor ( $0.4 \% \mathrm{ID} / \mathrm{g}$ ), probably resulting from the reduced drainage occurring in the tumor, and in the kidney ( $\sim .15 \% \mathrm{ID} / \mathrm{g}$ ) as the preferential clearance organ for the DTPA molecule. The uptake in the other tissue/organs can be considered as negative (Fig 1A). 


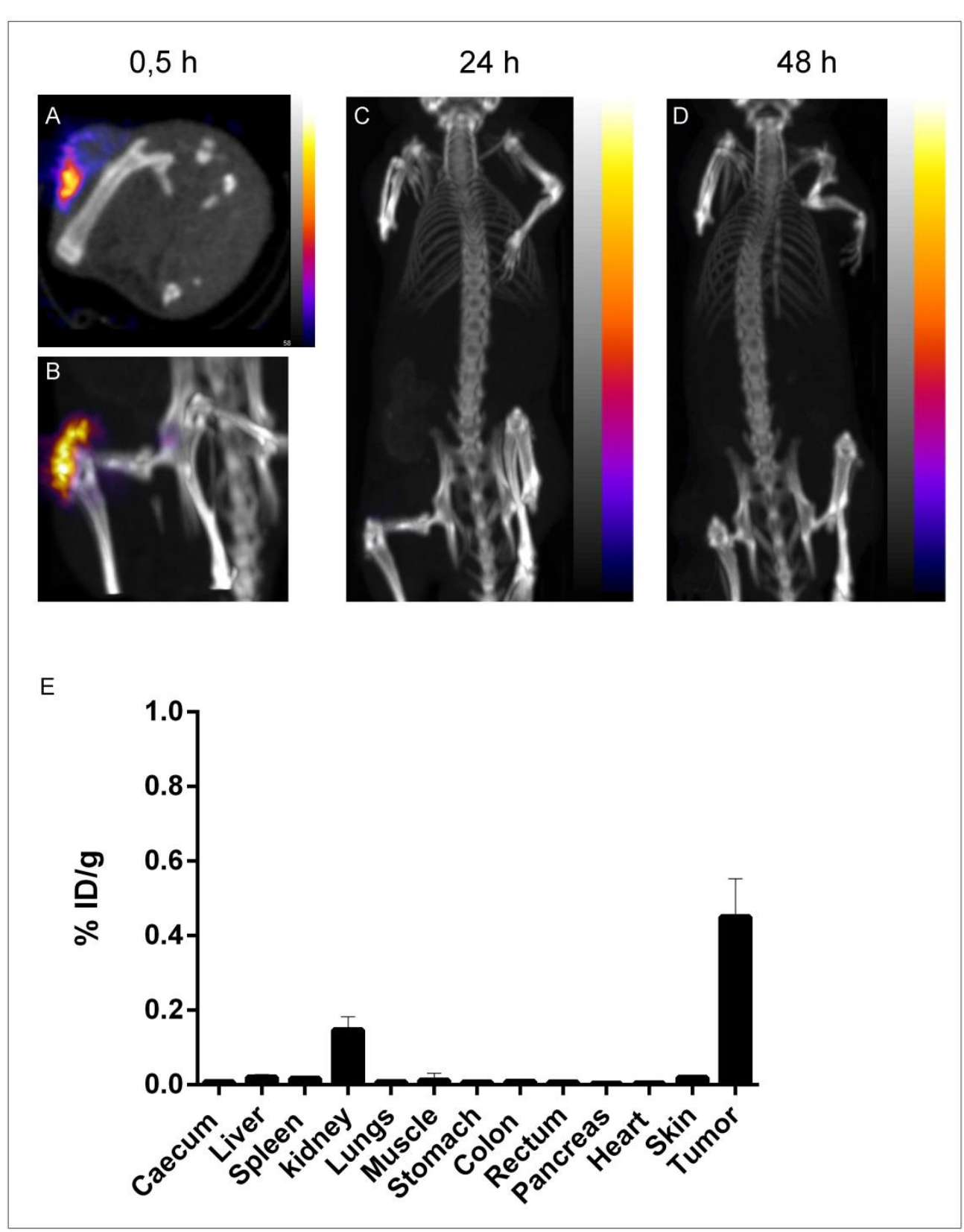

Fig 1. Result of negative control group. A-D) SPECT/CT images: A) Focus scan in the tumor region, transversal view ; B) Focus scan in the tumor region, maximum intensity projection; C-D) Whole body images, maximum intensity projection; E) Biodistribution data at $48 \mathrm{~h}$ pi. 